\title{
Development of the extra-thin UHF-RFID tag antenna for liquid containers identification
}

\author{
Atsuya Kadono $^{1, \text { a) }}$ and Yoshinobu Okano ${ }^{1, \text { b) }}$ \\ ${ }^{1}$ Tokyo City University, \\ 1-28-1 Tamadutsumi, Setagaya-Ku, Tokyo, 158-8557, Japan \\ a) g1981811@tcu.ac.jp \\ b)y-okano@tcu.ac.jp
}

Abstract: Recently, RFID (Radio Frequency IDentification) systems are expected to serve as the distribution management system of the alternative to bar-code systems. If tag antennas that can be identified even when attached to the surface of metal or high lossy dielectrics are developed, highly accurate automation of various product management can be expected. In this report, the development of the tag antenna for UHF-RFID system that can be attached to containers such as blood samples or liquid medicines is described.

Keywords: RFID, tag antenna, high lossy dielectrics

Classification: Small Antennas

\section{References}

[1] P.V. Nikitin, K.V.S. Rao, and S. Lazar, "An overview of near field UHF RFID," Proc. IEEE RFID Int. Conf., TX, pp. 167-174, March 2007. DOI: 10.1109/rfid. 2007.346165

[2] G. Marrocco, "RFID antennas for the UHF remote monitoring of human subjects," IEEE Trans. Antennas Propag., vol. 55, no. 6, pp. 1862-1870, June 2007. DOI: 10.1109/tap.2007.898626

[3] A. Taflove and M.E. Brodwin, "Numerical solution of steady-state electromagnetic scattering problem using the time-dependent Maxwell's equation," IEEE Trans. Microw. Theory Techn., vol. 23, no. 8, pp. 623-630, Aug. 1975. DOI: 10.1109/tmtt.1975.1128640

[4] K.S. Kunz and R.J. Lubbers, The finite difference time domain method for electromagnetics, CRC Press, Boca Raton, FL, 1993. DOI: 10.1201/9780203736708

[5] http://www.fcc.gov/fcc-bin/dielec.sh

[6] A. Kadono, Y. Toriya, S. Matsuoka, and Y. Okano, "Development of operational UHF-RFID tag antenna near the lossy liquid," 2019 International Workshop on Electromagnetics (iWEM), Qingdao, China, 2019. DOI: 10.1109/iwem. 2019.8887888

[7] S. Matsuoka, Y. Toriya, A. Kadono, and Y. Okano, "Study on objects storage position detector with UHF-RFID system," IEICE Commun. Express, vol. 8, no. 12, pp. 474-479, 2019. DOI: 10.1587/comex.2019gcl0011 


\section{Introduction}

RFID (Radio Frequency IDentification) systems are expected to serve as the distribution management system of the alternative to bar-code systems in recent years. Because RF-ID systems can perform multifaceted inspection and recognition with the non-contact manner by wireless technologies, it is widely used in the distribution management field [1]. Additionally, by developing a tag antenna that can be recognized even if attached to metal or high lossy dielectric surface, it enables highly accurate automation of various product management. Currently, there are two types of RFID systems (the electromagnetic coupling use type and the microwave-use type). The electromagnetic coupling use type system (HF-RFID) has the property to which the identification precision is steady for environmental changes. Therefore, this system is suitable for the detection of closely spaced RF-tags. However, it is unsuitable for few meters distant or movement RF-Tag detection. In contrast, the microwave-use type system (UHF-RFID) is suitable for few meters distant or traveling RF-tags detection, because the detection signal transmission efficiency is high [2]. However, the radiation property of UHF-RFID tag is strongly influenced with the adjoining dielectric substance. It has been widely used in the apparel industry because UHF-RFID-tags have above mentioned features. If tag antennas that can be identified even when attached to the surface of metal or high lossy dielectrics are developed, its use coverage will be expanded drastically. Consequently, the development of the tag-antenna for UHF-RFID system which can be attached to containers such as blood samples or liquid medicines is described in this report.

\section{Configuration of the proposed system}

The proposed tag antenna's structure and the tag connected RF-IC chip's (U-code8 manufactured by NXP semiconductors) equivalent circuit is shown in Fig. 1.

Tag antenna is composed of the metal ribbon looped like the rectangular ring along the acrylic resin square pillar. The RF-IC chip is loaded on the tag antenna's

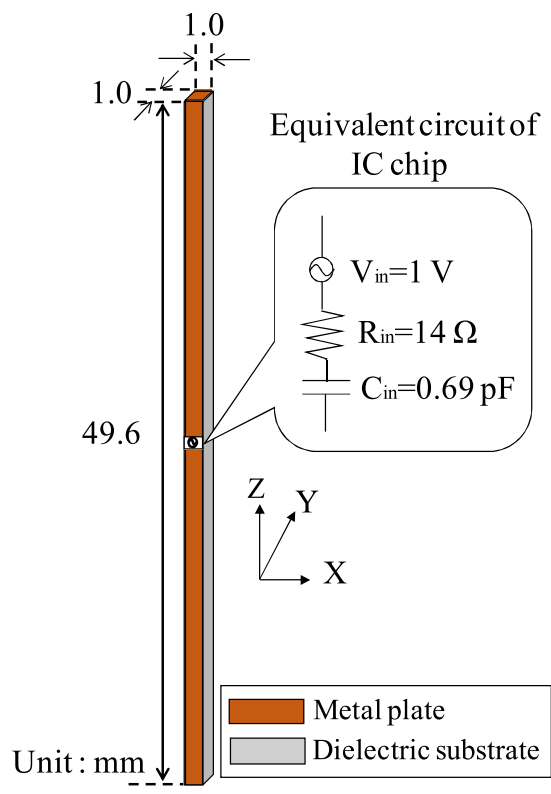


side. The depth and the width of the square pillar are set to $1.0 \mathrm{~mm}$, respectively. In addition, the length of square pillar is optimized as $49.6 \mathrm{~mm}$ with numerical analysis. Dielectric properties of the substrate (relative permittivity $\varepsilon_{\mathrm{r}}$ and the conductivity $\sigma$ ) which composes the tag antenna are set to $\varepsilon_{\mathrm{r}}=2.8$ and $\sigma=1.0 \times 10^{-10} \mathrm{~S} / \mathrm{m}$, respectively. The finite difference time domain (FDTD) method is used to the parametric study [3, 4]. The target frequency is set to $920 \mathrm{MHz}$, which is the center frequency of the UHF-RFID band. In addition, the requirement for VSWR is set to 2 or less.

\section{Simulated results}

In this chapter, analytical results of the radiation and input properties of the proposal antenna attached to the test tube filled with lossy liquid are described. The location of the tag antenna mounted on the test-tube filled with lossy liquid is shown in Fig. 2(a). In here, blood is assumed as the lossy liquid. The relative permittivity and the conductivity of the test tube are set to $\varepsilon_{\mathrm{r}}=4.8$ and $\sigma=1.0 \times 10^{-10} \mathrm{~S} / \mathrm{m}$ respectively. Also, the dielectric properties of blood are set to $\varepsilon_{\mathrm{r}}=61.3$ and $\sigma=1.6 \mathrm{~S} / \mathrm{m}$, respectively [5]. Analysis input property of tag antenna attached on the lossy liquid container is shown in Fig. 2(b). In this figure, $Z_{o}=R_{i n}=14 \Omega$ is set as the normalized impedance. In the past proposed thin loop antenna, the loop aperture side was faced with the test tube to minimize the influence of the lossy liquid [6, 7]. The null had appeared in the azimuth plane though the radiation efficiency was good (around $88 \%$ ) in that procedure. On the other hand, when the metal ribbon side is faced with the lossy liquid shown as Fig. 2(a), the antenna's band width has been expanded more than [6], though the radiation efficiency is deteriorated around $30 \%$. As result, it is confirmed that the requirement for tag antenna is achieved $($ VSWR $=1.10)$ at $920 \mathrm{MHz}$. The radiation properties at $920 \mathrm{MHz}$ with the tag antenna attached on the test tube are shown in Fig. 2(c). As a result of this figure, the tag antenna's radiation shows omnidirectional pattern in X-Y plane. In Y-Z plane, the radiation characteristics of the tag antenna show a low radiation gain in the $\mathrm{Z}$ direction, though the maximum antenna gain reaches $1.60 \mathrm{dBi}$. However, the average gain of this antenna in the $\mathrm{Y}-\mathrm{Z}$ plane reaches $-2.74 \mathrm{dBi}$. It is thought that this tag can respond enough to the tag identification signal which comes from an arbitrary direction in $\mathrm{X}-\mathrm{Y}$ plane.

\section{Measurement results}

In this chapter, experiment results with the manufactured prototype tag antenna are described. The prototype tag antenna is composed of the copper tape looped like the rectangular ring along the acrylic resin square pillar (see Fig. 3(a)).

The tag antenna is measured by attaching a test tube filled with blood phantom (relative permittivity $\varepsilon_{\mathrm{r}}=59.4$ and the conductivity $\sigma=1.7 \mathrm{~S} / \mathrm{m}$ ) as shown in Fig. 3(b). The gun-type Reader/Writer (BHT-1281QULWB-CE) is used for evaluation of the prototype tag (see Fig. 3(c)). The output of R/W is set by $100 \mathrm{~mW}$. Direction dependence of communicable distance at $920 \mathrm{MHz}$ with the prototype tag is shown in Fig. 3(d). From the measurement result, the communicable range is from $50 \mathrm{~cm}$ to $73 \mathrm{~cm}$, under the condition to set the antenna output to $100 \mathrm{~mW}$. It is 

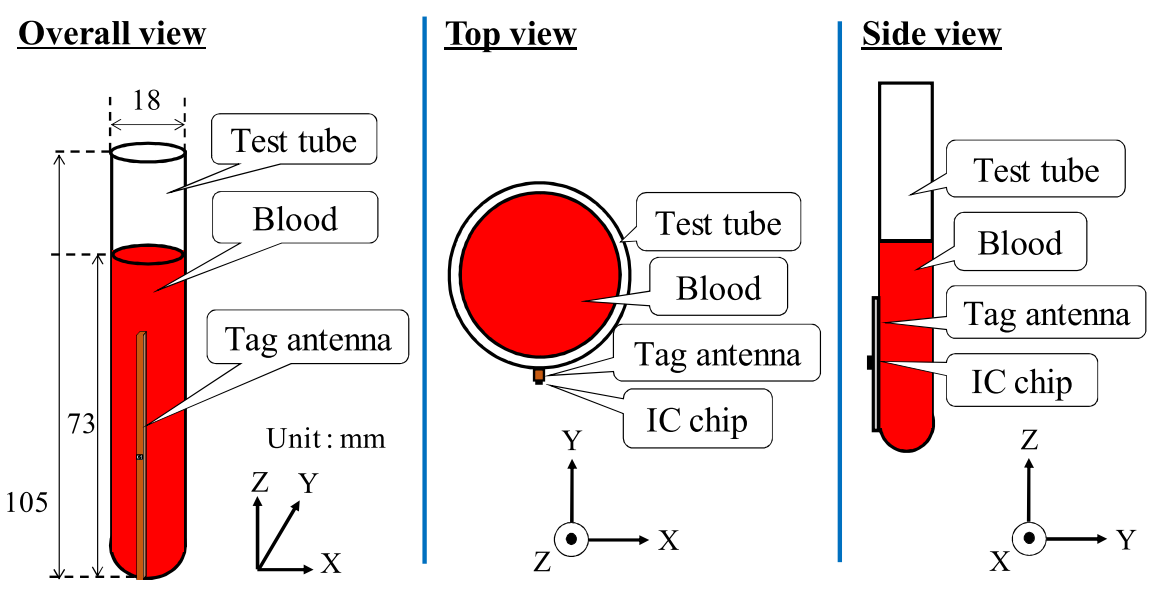

(a)

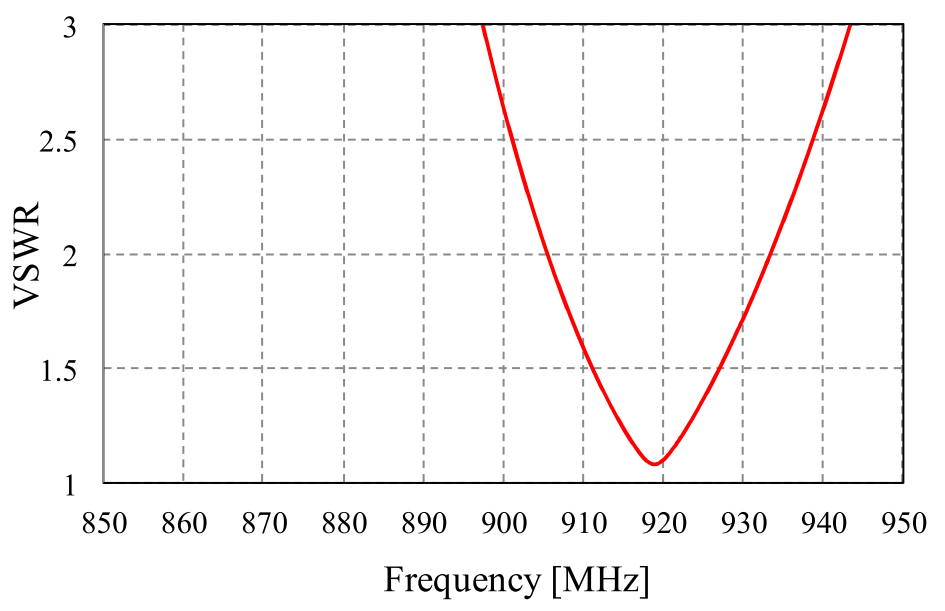

(b)
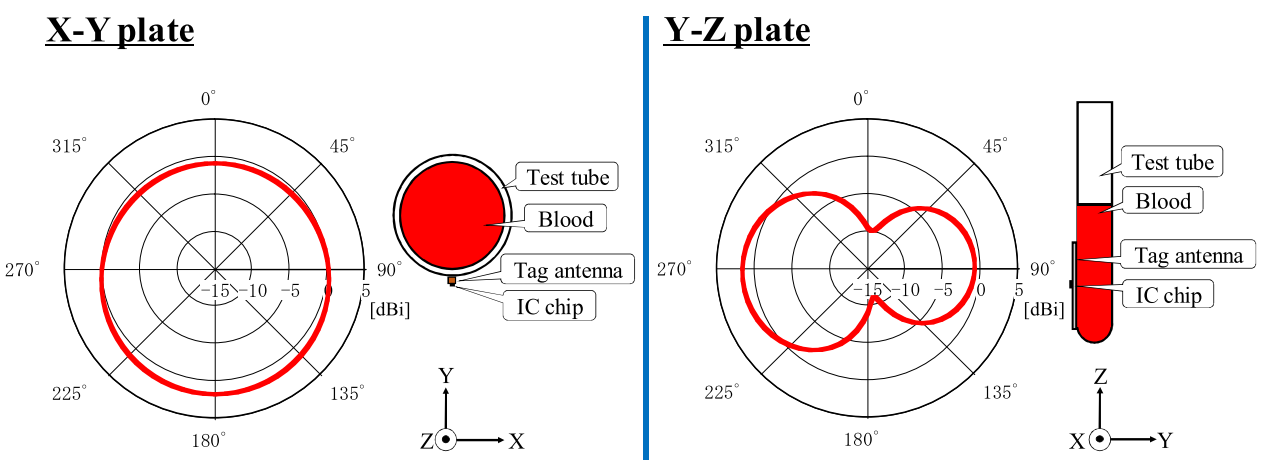

(c)

Fig. 2. Evaluation results of the proposed tag antenna. (a) Analysis model when tag antenna is attached to test tube filled with blood. (b) Analysis result of VSWR. (c) Analysis result of radiation pattern at $920 \mathrm{MHz}$.

confirmed that the proposal tag has enough communicable range under the limit of the low power wireless equipment, as these results. 

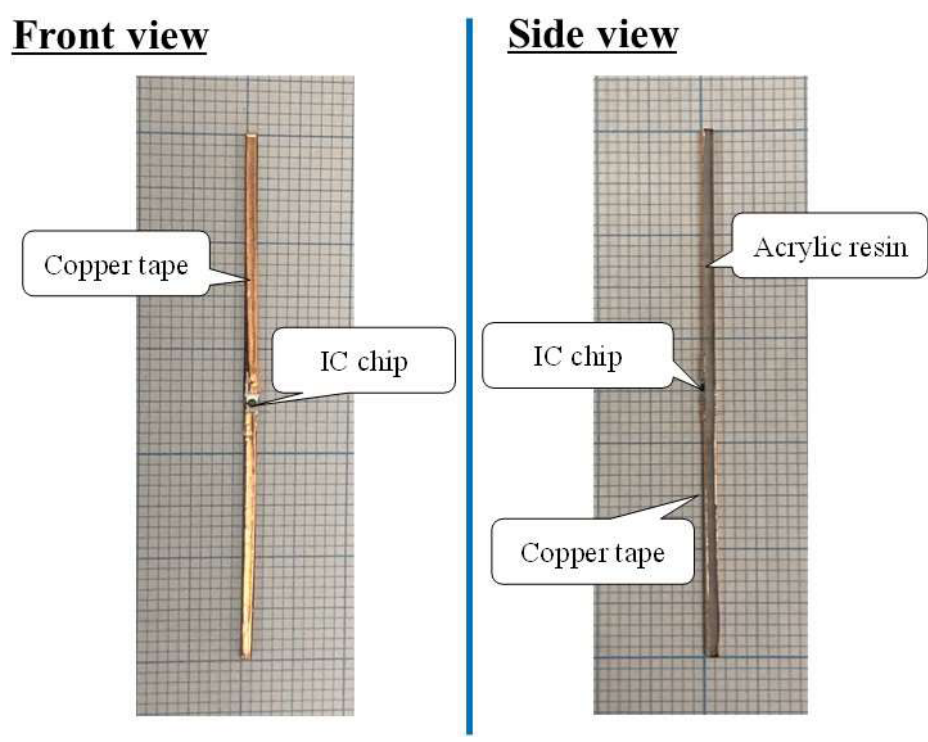

(a)
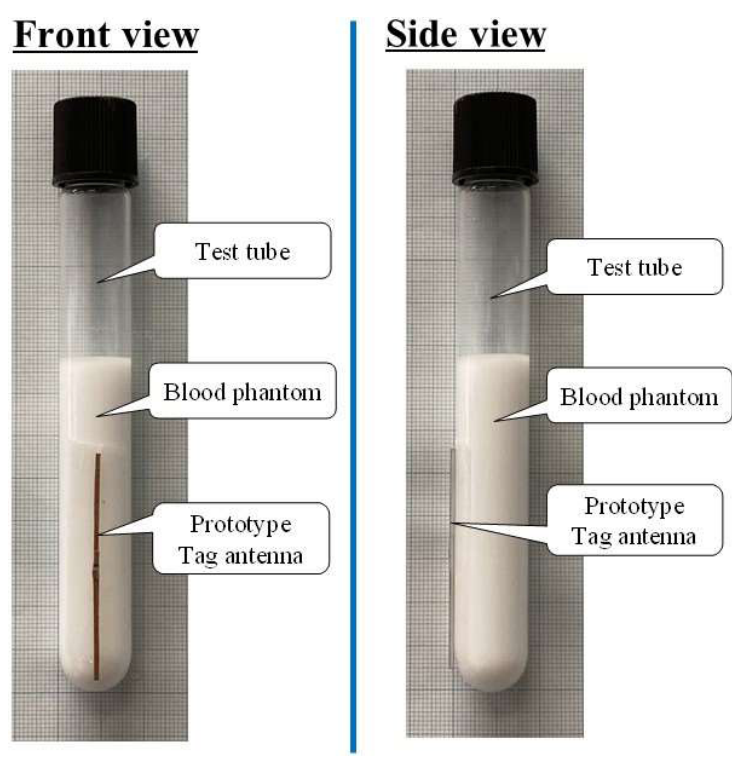

(b)

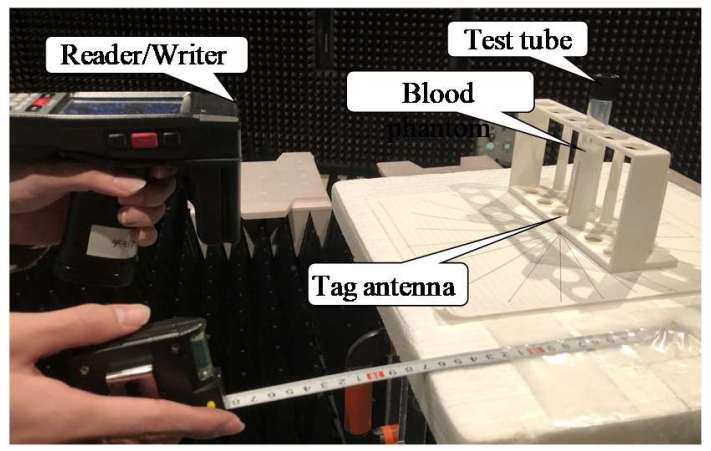

(c)

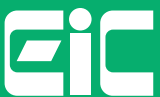

๑ IEICE 2021

DOI: 10.1587/comex.2021SPL0025

Received April 8, 2021

Accepted May 6, 2021

Publicized August 12, 2021

Copyedited September 1, 2021 


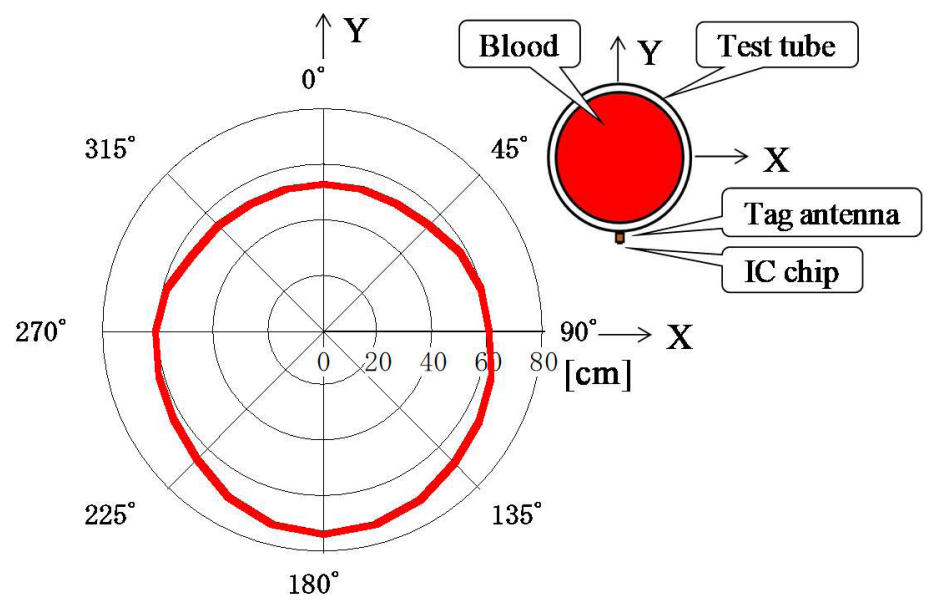

(d)

Fig. 3. Direction dependence of communications distance at $920 \mathrm{MHz}$. (a) The manufactured tag antenna. (b) The manufactured tag antenna attached to a test tube filled with blood. (c) Prototype tag's communicable range measurement scenery. (d) The measured communicable distance property at $920 \mathrm{MHz}$

\section{Conclusion}

In this report, the practicality of the UHF RFID tag antenna employable near the lossy liquid was verified. Concretely, the input and radiation properties with an extra-thin rectangular loop antenna attached on the test tube filled with blood were evaluated. As a result, in the simulation, it was confirmed that the extra-thin loop antenna demonstrated excellent properties near the lossy liquid like blood. Also, in the measurement result, it was confirmed that the proposal tag had enough communicable range under the limit of the low power wireless equipment. In the near future, the identification performance when proposal tags are loaded to the overcrowded high lossy dielectric samples will be evaluated. 